\title{
TITLE:
}

\section{Ab initio calculation of electron effective masses in solid pentacene}

$\operatorname{AUTHOR}(\mathrm{S})$ :

Doi, K; Yoshida, K; Nakano, H; Tachibana, A;

Tanabe, T; Kojima, Y; Okazaki, K

\section{CITATION:}

Doi, K ...[et al]. Ab initio calculation of electron effective masses in solid pentacene. JOURNAL OF APPLIED PHYSICS 2005, 98(11): 113709.

\section{ISSUE DATE:}

2005-12-01

URL:

http://hdl.handle.net/2433/39718

\section{RIGHT:}

Copyright 2005 American Institute of Physics. This article may be downloaded for personal use only. Any other use requires prior permission of the author and the American Institute of Physics. 


\title{
$A b$ initio calculation of electron effective masses in solid pentacene
}

\author{
K. Doi, K. Yoshida, H. Nakano, and A. Tachibana ${ }^{a}$ \\ Department of Microengineering, Kyoto University, Kyoto 606-8501, Japan \\ T. Tanabe \\ Pioneer Corporation, 6-1-1 Fujimi, Tsurugasaki, Saitama 350-2288, Japan \\ Y. Kojima and K. Okazaki \\ MCC-Group Science and Technology Research Center, Mitsubishi Chemical Corporation, 1, Toho-cho, \\ Yokkaichi, Mie 510-8530, Japan
}

(Received 23 March 2005; accepted 24 October 2005; published online 9 December 2005)

\begin{abstract}
We have calculated the band structures of solid pentacene. One of our models makes use of experimental data obtained for a single crystal [R. B. Campbell et al., Acta. Crystallogr. 14, 705 (1961); R. B. Campbell and J. M. Robertson, Acta. Crystallogr. 15, 289 (1962)] and the other one considers a thin film fabricated by the present authors. Our calculations indicate that the electronic properties of a pentacene thin film are markedly different from those of a single crystal, although the densities of pentacene molecules in these solids are not significantly different. The energy band gaps of the single crystal and the thin film have been estimated to be $0.73 \mathrm{eV}$ and between 0.26 and $0.72 \mathrm{eV}$, respectively. The bandwidths of the highest valence band and the lowest conduction band of the film are wider than those of the single crystal. These results suggest that the electron or hole carriers in the film have effective masses lighter than those of the single crystal, and in order to estimate the carrier mobility we first calculate the effective mass. (C) 2005 American Institute of Physics. [DOI: 10.1063/1.2138381]
\end{abstract}

\section{INTRODUCTION}

Recently, interest in applications of organic semiconductors has increased in both academic and industrial material research because of their advantageous characteristics such as flexibility, low cost, lightweight, and nontoxicity. Some organic molecular crystals and thin films have received much attention due to their great potential for application to modern electronic devices, and numerous reports on highmobility organic thin films have been published. ${ }^{1-6}$ In particular, pentacene $\left(\mathrm{C}_{22} \mathrm{H}_{14}\right)$ thin films have been one of a number of remarkable materials focused on in recent research on organic molecular solids. Dimitrakopoulos et al. reported that the structure of pentacene films is different from that of the single crystals and that pentacene thin films are stable and have a high electron mobility at room temperature. ${ }^{1}$ Recent research on organic thin-film transistors is reviewed in Ref. 2. Minakata and co-workers investigated some interesting properties of iodine-doped pentacene films and reported that the long axes of iodine-doped pentacene molecules were aligned perpendicular to the substrate plane, and the films had a conduction plane parallel to the substrate and had very low conductivity along the vertical direction. ${ }^{7-9}$

Generally, charge transport in organic crystals depends on carrier hopping at high temperatures and on band-type conduction at low temperatures. ${ }^{10}$ Kenkre et al. calculated the electron mobilities of naphthalene crystal and simulated the dependency of electron-phonon coupling in charge transfer: the mobilities deduced from their model Hamiltonian showed a substantial agreement with the experimental data. ${ }^{11}$

\footnotetext{
a) Author to whom correspondence should be addressed; FAX: +81-75-7535184; electronic mail: akitomo@scl.kyoto-u.ac.jp
}

Silinsh and co-workers investigated polaronic states in a number of molecular crystals in detail. ${ }^{12-14}$ Recently, Tiago et al. have carried out an ab initio study of solid pentacene and discussed its electronic and optical properties. ${ }^{15}$ However, the charge transport mechanism of solid pentacene has not yet been clarified completely, although many theoretical models have been proposed and agreement between these models and experimental data obtained for a number of molecular crystals has been achieved. In this paper, we report the results of $a b$ initio calculations of electronic band structures and electron effective masses in pentacene solids performed on the basis of density-functional theory and discuss the electronic properties in terms of quantum energy density. ${ }^{16-23}$

\section{COMPUTATIONAL METHOD}

We carried out the energy-band calculations for periodic models of solid pentacene. One of the models is of a pentacene single crystal and makes use of experimental data. ${ }^{24,25}$ The other is a model of a pentacene thin film that we previously fabricated. ${ }^{26}$ Figure 1 shows the two models of solid pentacene, the unit cells of which are composed of two pentacene molecules. The single-crystal model is a triclinic system where the cell parameters, as taken from Ref. 24 , are $a=7.93 \AA, b=6.14 \AA$, and $c=16.03 \AA$, and $\alpha=101.9^{\circ}, \beta$ $=112.6^{\circ}$, and $\gamma=85.8^{\circ}$ as shown in Fig. 1(a). The thin-film model is based on our original sample, the cell parameters of which were derived by x-ray-diffraction analysis as $a$ $=7.50 \AA, b=5.92 \AA$, and $c=15.35 \AA$, and $\alpha=90.0^{\circ}, \beta$ $=90.0^{\circ}$, and $\gamma=91.0^{\circ} .{ }^{26}$ In this work, we considered the imaginary models of a pentacene thin film because our experimental instrument could not accurately determine the ori- 


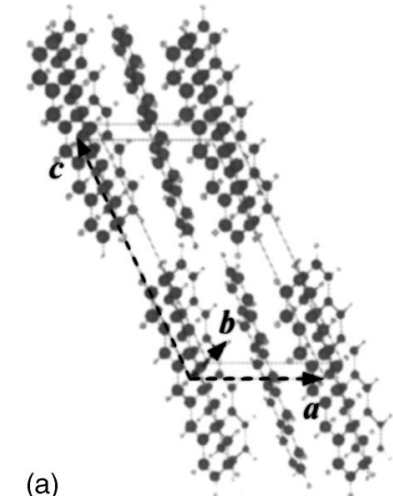

(b)

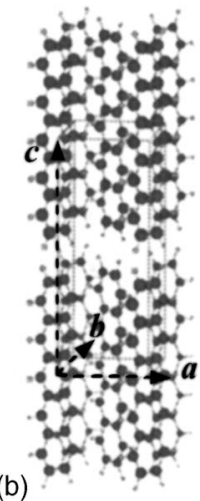

FIG. 1. (a) Unit cell of pentacene single crystal whose parameters are $a$

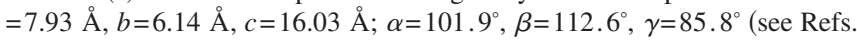
24 and 25), and (b) unit cell of pentacene thin film whose parameters are $a=7.50 \AA, b=5.92 \AA, c=15.35 \AA ; \alpha=90.0^{\circ}, \beta=90.0^{\circ}$, and $\gamma=91.0^{\circ}$ (see Ref. 26).

entations of the molecules relative to the plane of the substrate. In our computations, the atomic positions were relaxed and optimized in the unit cells before calculating the band structures. The electron density, under a periodic boundary condition, was calculated using density-functional theory (DFT) with a periodic regional DFT (PRDFT) program package. $^{27}$ The norm-conserving pseudopotential $^{28}$ and generalized-gradient approximation functional formulated by Perdew and Wang ${ }^{29}$ and Perdew and co-workers ${ }^{30}$ were employed. Electron wave functions were expanded using plane waves and adopted up to $1088 \mathrm{eV}$. Figure 2 shows the first Brillouin zones and reciprocal lattice vectors for the unit cells shown in Fig. 1.

We calculated the electronic energy bands along the reciprocal lattice vectors and several specific paths on the boundary surfaces of the first Brillouin zone, and derived the electron effective masses at certain points in the momentum space. The effective mass tensor can be estimated by

$$
\frac{1}{m^{*}}=\left(\begin{array}{ccc}
\frac{\partial^{2} \epsilon}{\partial k_{x}^{2}} & \frac{\partial^{2} \epsilon}{\partial k_{x} \partial k_{y}} & \frac{\partial^{2} \epsilon}{\partial k_{x} \partial k_{z}} \\
\frac{\partial^{2} \epsilon}{\partial k_{y} \partial k_{x}} & \frac{\partial^{2} \epsilon}{\partial k_{y}^{2}} & \frac{\partial^{2} \epsilon}{\partial k_{y} \partial k_{z}} \\
\frac{\partial^{2} \epsilon}{\partial k_{z} \partial k_{x}} & \frac{\partial^{2} \epsilon}{\partial k_{z} \partial k_{y}} & \frac{\partial^{2} \epsilon}{\partial k_{z}^{2}}
\end{array}\right),
$$

where $\epsilon$ is an eigenvalue at a $k$ point. ${ }^{31}$ In this study, the derivatives in Eq. (1) were calculated using a finitedifference approximation, (a)

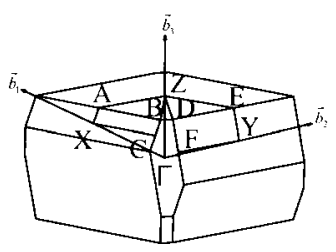

(b)

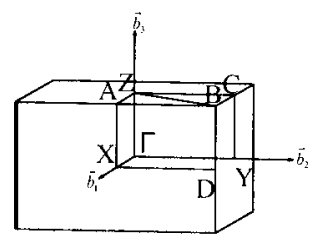

FIG. 2. Reciprocal lattice vectors and first Brillouin zone of solid pentacene: (a) pentacene single crystal shown in Fig. 1(a), and (b) pentacene thin film shown in Fig. 1(b).

$$
\begin{aligned}
\frac{\partial^{2} \epsilon}{\partial k_{i}^{2}}= & \frac{1}{\left(\Delta k_{i}\right)^{2}}\left[\epsilon\left(k_{i}+\Delta k_{i}, k_{j}, k_{k}\right)-2 \epsilon\left(k_{i}, k_{j}, k_{k}\right)\right. \\
+ & \left.\epsilon\left(k_{i}-\Delta k_{i}, k_{j}, k_{k}\right)\right], \\
\frac{\partial^{2} \epsilon}{\partial k_{i} \partial k_{j}}= & \frac{1}{\left(2 \Delta k_{i}\right)\left(2 \Delta k_{j}\right)}\left[\epsilon\left(k_{i}+\Delta k_{i}, k_{j}+\Delta k_{j}, k_{k}\right)\right. \\
& -\epsilon\left(k_{i}+\Delta k_{i}, k_{j}-\Delta k_{j}, k_{k}\right) \\
& -\epsilon\left(k_{i}-\Delta k_{i}, k_{j}+\Delta k_{j}, k_{k}\right) \\
& \left.+\epsilon\left(k_{i}-\Delta k_{i}, k_{j}-\Delta k_{j}, k_{k}\right)\right],
\end{aligned}
$$

where $\{i, j, k\}=\{x, y, z\}$ and $\Delta k_{i}=\Delta k_{j}=\Delta k_{k}=0.044\left(\right.$ a.u. $\left.^{-1}\right)$. Diagonalized effective masses and their corresponding eigenvectors are shown.

Kinetic-energy density $n_{T}(\vec{r})$, tension density $\vec{\tau}^{S}(\vec{r})$, and stress tensor density $\overleftrightarrow{\tau}^{S}(\vec{r})$, which were derived in Refs. 16-23, were calculated from electron wave functions. Kinetic-energy density $n_{T}(\vec{r})$ is represented by

$$
n_{T}(\vec{r})=-\frac{\hbar^{2}}{4 m} \sum_{i} \nu_{i}\left[\psi_{i}^{*}(\vec{r}) \Delta \psi_{i}(\vec{r})+\Delta \psi_{i}^{*}(\vec{r}) \psi_{i}(\vec{r})\right],
$$

where $\nu_{i}$ is an occupation number of the $i$ th state. The electronic motion obeys classical mechanics in the electronic drop region $R_{D}$ where $n_{T}(\vec{r})>0$ and cannot be explained without quantum mechanics in the electronic atmosphere region $R_{A}$ where $n_{T}(\vec{r})<0$. The boundary between $R_{D}$ and $R_{A}$ is the turning point of electronic motion and is called the electronic interface $S .^{16-23}$

A component of tension density $\vec{\tau}^{S}(\vec{r})$ is given by

$$
\begin{aligned}
\tau^{S k}(\vec{r})= & \frac{\hbar^{2}}{4 m} \sum_{i} \nu_{i}\left[\psi_{i}^{*}(\vec{r}) \frac{\partial \Delta \psi_{i}(\vec{r})}{\partial x^{k}}-\frac{\partial \psi_{i}^{*}(\vec{r})}{\partial x^{k}} \Delta \psi_{i}(\vec{r})\right. \\
& \left.+\frac{\partial \Delta \psi_{i}^{*}(\vec{r})}{\partial x^{k}} \psi_{i}(\vec{r})-\Delta \psi_{i}^{*}(\vec{r}) \frac{\partial \psi_{i}(\vec{r})}{\partial x^{k}}\right],
\end{aligned}
$$

and in the stationary state, this force density from the kineticenergy density is in balance with another force density from the potential-energy density. An element of stress tensor density $\overleftrightarrow{\tau}^{S}(\vec{r})$ is represented by

$$
\begin{aligned}
\tau^{S k l}(\vec{r})= & \frac{\hbar^{2}}{4 m} \sum_{i} \nu_{i}\left[\psi_{i}^{*}(\vec{r}) \frac{\partial^{2} \psi_{i}(\vec{r})}{\partial x^{k} \partial x^{l}}-\frac{\partial \psi_{i}^{*}(\vec{r})}{\partial x^{k}} \frac{\partial \psi_{i}(\vec{r})}{\partial x^{l}}\right. \\
& \left.+\frac{\partial^{2} \psi_{i}^{*}(\vec{r})}{\partial x^{k} \partial x^{l}} \psi_{i}(\vec{r})-\frac{\partial \psi_{i}^{*}(\vec{r})}{\partial x^{l}} \frac{\partial \psi_{i}(\vec{r})}{\partial x^{k}}\right] .
\end{aligned}
$$

This tensor density shows the properties of the chemical bonds in detail, as shown in Ref. 21-23. In this study, we diagonalized $\overleftrightarrow{\tau} S(\vec{r})$ and show here the largest eigenvalues and their eigenvectors at each $\vec{r}$. The set of the largest eigenvalues of $\overleftrightarrow{\tau}^{S}(\vec{r})$ can represent covalent bonds, such as $\mathrm{C}-\mathrm{C}$ bonds, formed by tensile stress, which resemble a "spindle structure." On the other hand, ionic bonds or metallic bonds can be represented in terms of compressive stress. In Ref. 21, images are shown for a number of samples in which the tensile stress appearing on $\mathrm{C}-\mathrm{C}$ covalent bonds and compressive stress on atoms are clearly represented. These densities can be originally calculated using the PRDFT program 


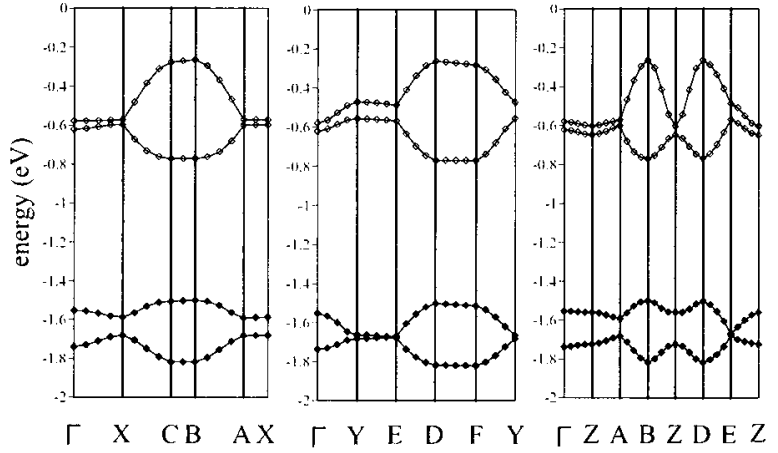

FIG. 3. Band structures of pentacene single crystal. The symmetry points and paths in the first Brillouin zone are shown in Fig. 2(a). The highest two valence bands and the lowest two conduction bands are, respectively, plotted with closed and open diamonds.

package, ${ }^{27}$ and we have demonstrated the usefulness of these densities in the analysis of computational results.

\section{RESULTS AND DISCUSSION}

Figures 3 and 4 show the energy-band structures of a single crystal and thin film of pentacene. The highest two valence bands and the lowest two conduction bands are plotted with closed and open diamonds, respectively. On the basis of these band structures, it has been clarified that the marked differences exist between the single crystal and thin film. Energy gaps $\left(E_{g} s\right)$ of $0.93,0.73,0.73,0.73$, and $0.74 \mathrm{eV}$ at $\Gamma, B, C, D$, and $F$ points, respectively, were obtained for the gap between the highest valence band and the lowest conduction band in the crystal, and, for a direct gap, $E_{g}$ between the top of the valence band and the bottom of the conduction band was $0.73 \mathrm{eV}$. On the other hand, $E_{g} s$ was 0.47 and $0.55 \mathrm{eV}$ in the thin film at $\Gamma$ and $Z$ points, respectively, and, for an indirect gap, $E_{g}$ between the top of the valence band and the bottom of the conduction band was $0.44 \mathrm{eV}$. It was clarified that $E_{g}$ in the crystal is larger than that in the film, although these gaps are underestimated by density-functional theory. The bandwidths of the highest valence band and the lowest conduction band were 0.17 and $0.21 \mathrm{eV}$ in the single crystal and 0.56 and $0.33 \mathrm{eV}$ in our thin film, respectively. These results imply that the band curvatures around certain states in the thin film are steeper and the effective masses of charge carriers in this film are lower than

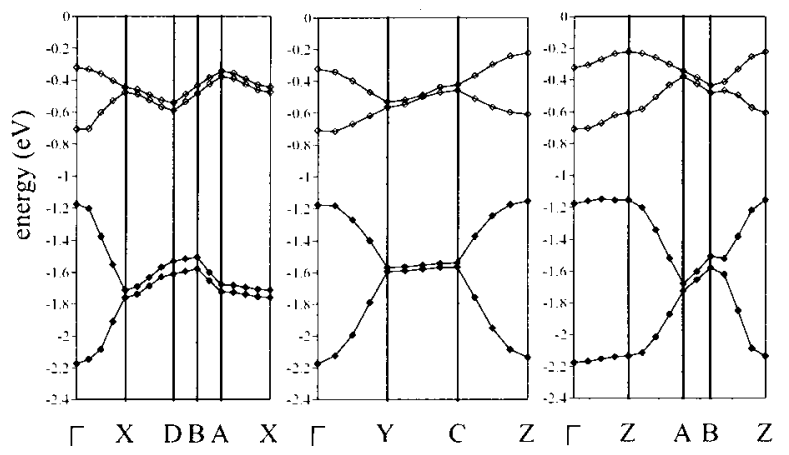

FIG. 4. Band structures of pentacene thin film. The symmetry points and paths in the Brillouin zone are shown in Fig. 2(b). Closed and open diamonds denote the same as those in Fig. 3.

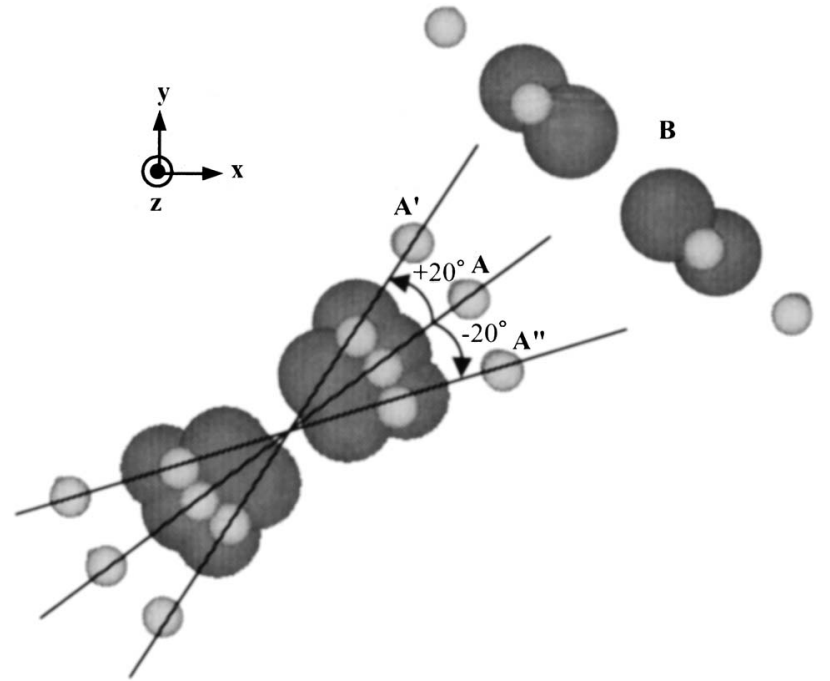

FIG. 5. There are two pentacene molecules named $A$ and $B$ in the unit cell of the thin film. The molecules $A^{\prime}$ and $A^{\prime \prime}$ are, respectively, in locations corresponding to the molecule $A$ being rotated by $+20^{\circ}$ and $-20^{\circ}$ around its long axis. Hereafter, the orientations of these molecules, $A$ and $B, A^{\prime}$ and $B$, and $A^{\prime \prime}$ and $B$, are called $A B, A^{\prime} B$, and $A^{\prime \prime} B$ orientations, respectively.

those in the single crystal. Our results of the band calculations based on the experimental data in Refs. 24 and 25 agrees with the computational results of Tiago et al. ${ }^{15}$ Their results also show that the band gap is between 0.7 and $0.8 \mathrm{eV}$ and the bandwidth of $\Gamma-Z$ interval is very narrow for the polymorphism.

Next, we examined the same calculation described above for the other two models to investigate electronic properties of our thin film in detail. The orientation of pentacene molecules in the $a b$ plane parallel to the substrate has not been clarified by our experiment and various orientations of molecules in this plane should be considered. Two pentacene molecules in a unit cell of the thin film are named $A$ and $B$; hereafter, we call the orientation of these two molecules the $A B$ orientation. Figure 5 shows molecules $A$ and $B$, and two other molecules named $A^{\prime}$ and $A^{\prime \prime}$ which are in the positions corresponding to $A$ being rotated by $+20^{\circ}$ and $-20^{\circ}$, respectively, around its long axis. The total energies of these three orientations, $A B, A^{\prime} B$, and $A^{\prime \prime} B$, are $-268.798,-268.796$, and -268.725 hartree, respectively. The $A B$ orientation was the most stable in the thin film models but its total energy was higher than that of the single crystal, -268.863 hartree. The $A^{\prime \prime} B$ orientation was not as stable as the $A B$ and $A^{\prime} B$ orientations. This result implies that pentacene molecules in the film prefer to orient in a herringbone structure rather than be oriented parallel to each other. We carried out band calculations for these film models in the same way as described above. The band structure of the $A^{\prime} B$ orientation is shown in Fig. 6, and $E_{g} s$ was $0.87,0.96$, and $0.74 \mathrm{eV}$ at $\Gamma, Z$, and $D$ points, respectively. In this case, the values of $E_{g} s$ were larger than those for the $A B$ orientation and $E_{g}$ between the top of the valence band and the bottom of the conduction band was $0.72 \mathrm{eV}$, showing an indirect gap. While in the case of the $A^{\prime \prime} B$ orientation, as shown in Fig. 7, $E_{g} s$ was 0.30 and $0.37 \mathrm{eV}$ at $\Gamma$ and $Z$ points, respectively. The values of $E_{g} s$ became smaller than those for the $A B$ orientation and $E_{g}$ 


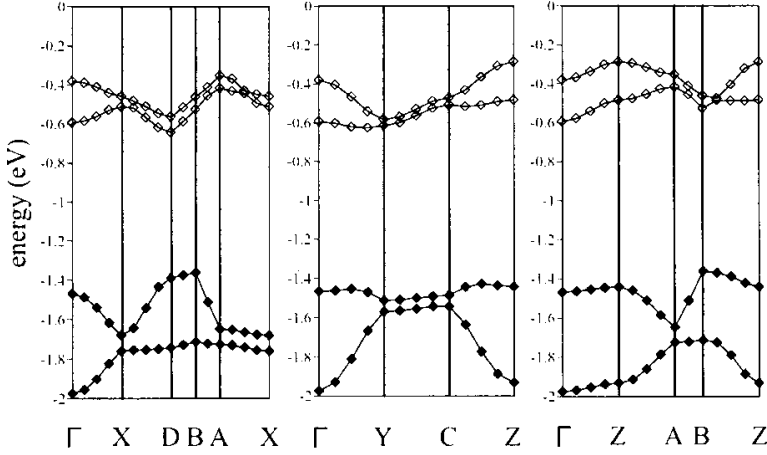

FIG. 6. Band structures of pentacene thin film in $A^{\prime} B$ orientation. Closed and open diamonds denote the same as those in Fig. 3.

between the top of the valence band and the bottom of the conduction band was $0.26 \mathrm{eV}$, showing an indirect gap. It was found that these band structures are clearly influenced by the orientation of pentacene molecules in the $a b$ plane, and these differences must affect parameters such as the electron effective mass and carrier mobility.

Table I shows diagonalized effective masses and eigenvectors calculated using Eqs. (1)-(3) for the single crystal ${ }^{24}$ and our thin-film models. The effective masses of the top of the valence band and the bottom of the conduction band were calculated at the $\Gamma$ and $B$ points in the single crystal and at the $\Gamma$ and $Z$ points in the films. The masses of a free electron and hole are 1.0 and -1.0 , respectively, in atomic units (a.u.). Our results shown in Table I indicate that the effective masses of carriers in the single crystal are higher than those of the free carriers, and thus carrier conduction is suppressed by the high mass. Carriers in the thin film, however, have low masses in $A B$ and $A^{\prime \prime} B$ orientations, especially along the $x$ and $z$ axes, where the $x y$ plane is parallel to the $a b$ plane and the $z$ axis is parallel to the $c$ axis. While in the $A^{\prime} B$ orientation, carriers usually tend to have high masses at the top of the valence band. It is concluded that the orientations of pentacene molecules clearly cause the differences in carrier mass not only in the $a b$ plane but also along the $c$ axis, and these different masses are expected to influence the carrier mobility $e \tau / m^{*}{ }^{32}$ where $\tau$ is the relaxation time. The low mass, however, does not directly correspond to a high mobility because impurities and phonon scatterings suppress

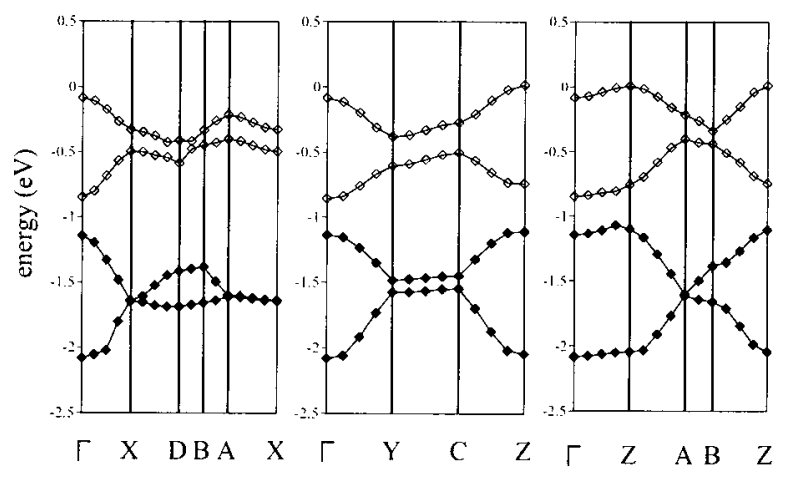

FIG. 7. Band structures of pentacene thin film in $A^{\prime \prime} B$ orientation. Closed and open diamonds denote the same as those in Fig. 3.
TABLE I. Eigenvalues of diagonalized effective masses and corresponding eigenvectors. Masses of a free electron and hole are, respectively, 1.0 and -1.0 in atomic units (a.u.).

\begin{tabular}{|c|c|c|c|c|c|c|c|c|}
\hline & \multicolumn{4}{|c|}{ Valence band top } & \multicolumn{4}{|c|}{ Conduction band bottom } \\
\hline & $m^{*}$ & $x$ & $y$ & $z$ & $m^{*}$ & $x$ & $y$ & $z$ \\
\hline \multicolumn{9}{|c|}{ Single crystal } \\
\hline \multirow[t]{3}{*}{$\Gamma$} & -5.6 & 0.32 & 0.58 & -0.75 & -4.3 & 0.69 & 0.66 & 0.30 \\
\hline & -3.1 & -0.69 & 0.68 & 0.23 & -3.0 & -0.22 & -0.20 & 0.96 \\
\hline & 48.0 & 0.65 & 0.45 & 0.62 & 1.9 & -0.69 & 0.72 & -0.01 \\
\hline \multirow[t]{3}{*}{$B$} & -16.3 & 0.09 & 0.72 & 0.69 & -11.6 & -0.17 & -0.13 & 0.98 \\
\hline & -5.4 & 0.01 & 0.69 & -0.72 & 2.2 & 0.98 & -0.07 & 0.16 \\
\hline & -2.6 & -1.00 & 0.07 & 0.05 & 13.3 & -0.04 & -0.99 & -0.14 \\
\hline
\end{tabular}

Thin film (A"B orientation)

$\begin{array}{rrrrrrrrr}\Gamma & -84.5 & 0.15 & 0.98 & -0.09 & 0.7 & 0.77 & -0.12 & 0.63 \\ & -0.6 & -0.99 & 0.15 & -0.01 & 0.7 & 0.63 & -0.05 & -0.77 \\ & 2.3 & 0.00 & 0.09 & 1.00 & 16.7 & -0.13 & -0.99 & -0.04 \\ Z & -2.4 & 0.18 & 0.98 & 0.00 & -0.1 & 0.00 & 0.00 & -1.00 \\ & -0.6 & 0.98 & -0.18 & 0.00 & 0.7 & -0.99 & 0.16 & 0.00 \\ & -0.1 & 0.00 & 0.00 & -1.00 & 2.5 & -0.16 & -0.99 & 0.00\end{array}$

Thin film ( $A B$ orientation)

$\begin{array}{rrrrr}\Gamma & -2.9 & -0.02 & 1.00 & -0.02 \\ & -0.4 & 0.01 & -0.02 & -1.00 \\ & -0.2 & 1.00 & 0.02 & 0.00 \\ & -3.4 & -0.01 & -0.07 & -1.00 \\ & -3.1 & 0.06 & -1.00 & 0.07 \\ & -0.7 & -1.00 & -0.06 & 0.01\end{array}$

$\begin{array}{rrrr}0.3 & -0.06 & -0.01 & 1.00 \\ 0.3 & 1.00 & 0.01 & -0.06 \\ 5.4 & 0.01 & -1.00 & 0.01 \\ -0.7 & 0.00 & 0.00 & -1.00 \\ 1.3 & 1.00 & 0.06 & 0.00 \\ 5.9 & 0.06 & -1.00 & 0.00\end{array}$

Thin film ( $A^{\prime} B$ orientation)

\begin{tabular}{rrrrrrrrr}
$\Gamma$ & -1.7 & -0.90 & 0.43 & 0.06 & -6.2 & 0.00 & -1.00 & 0.01 \\
& 2.8 & -0.14 & -0.16 & -0.98 & 0.7 & -0.03 & 0.01 & 1.00 \\
& 4.1 & -0.41 & -0.89 & 0.20 & 4.6 & -1.00 & 0.00 & -0.03 \\
$Z$ & -3.0 & -0.01 & 0.02 & -1.00 & -6.7 & 0.11 & 0.99 & -0.01 \\
& -1.7 & -0.90 & 0.44 & 0.02 & -0.6 & 0.00 & -0.01 & -1.00 \\
& 4.1 & -0.44 & -0.90 & -0.01 & 5.8 & 0.99 & -0.11 & 0.00 \\
\hline
\end{tabular}

carrier conduction and these effects are usually contained in $\tau$. Therefore, the carrier mobility is not simply proportional to the inverse of the effective mass as shown in Ref. 30.

We have calculated the electron densities and electron energy densities to discuss the reason why some carriers have lower effective masses in thin films than in a single crystal. Figures 8(a) and 8(b) show the electron densities, $8(\mathrm{c})$ and $8(\mathrm{~d})$ the kinetic-energy densities and the tension densities, and 8(e) and 8(f) the stress tensor densities and their eigenvectors in the single crystal. Figure 9 shows the electron densities and electron energy densities in the thin film in the same way as in Fig. 8. The electron densities and the kinetic-energy densities shown in Figs. 8 and 9 are those of the highest occupied valence band. These figures represent electrons in $\pi$ orbitals localized on pentacene molecules. The electrons on each molecule cannot cross to another molecule according to classical mechanics because the electronic drop region $R_{D}$ where electrons can move freely according to classical mechanics is closed on a pentacene molecule and there is a potential wall between the molecules, which cannot be overcome by an electron without the quantum tunneling effect. Furthermore, the tension density shows the direction of the force caused by the kinetic-energy density and this force density is in balance with the force from the potential-energy 

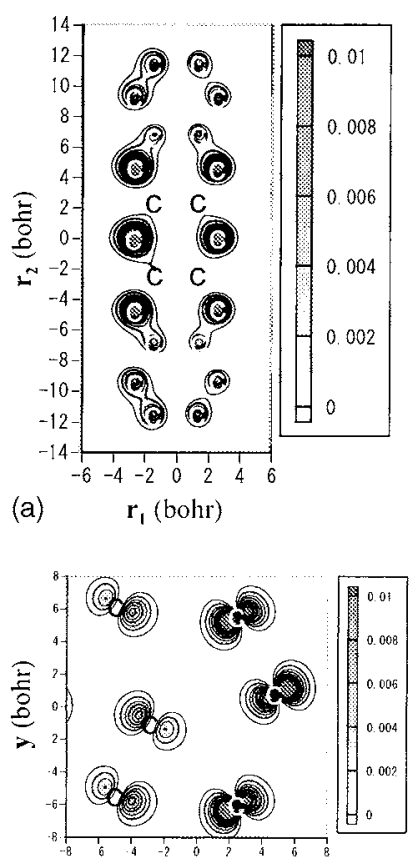

(b)

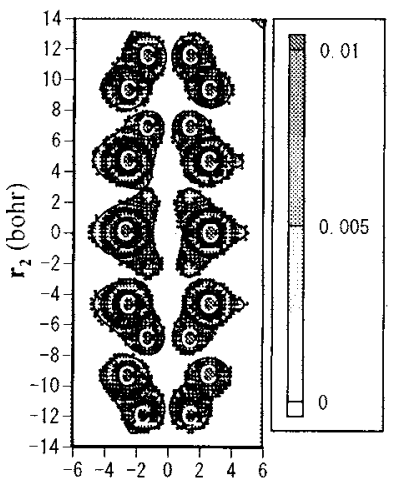

(c)

$\mathbf{r}_{1}$ (bohr)

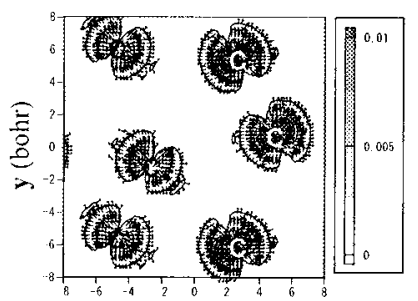

(d)

$\mathbf{x}$ (bohr)

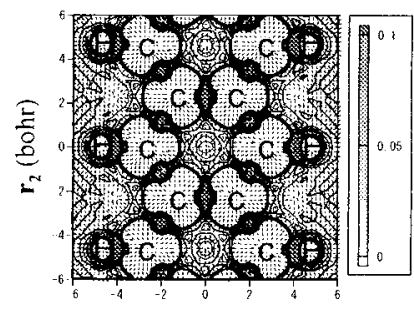

(e) $\quad \mathbf{r}_{1}$ (bohr)

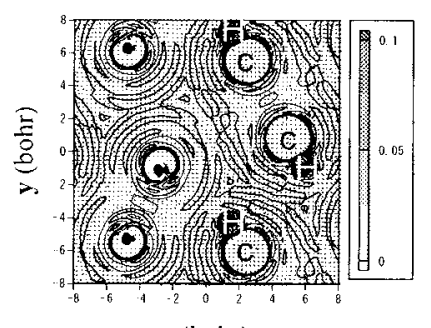

(f)

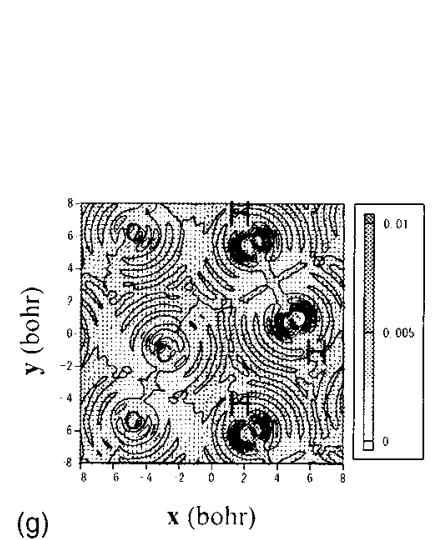

(g)

(c)

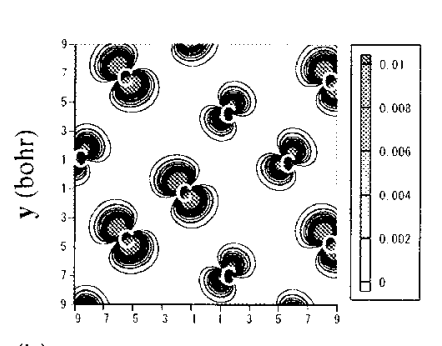

(b)

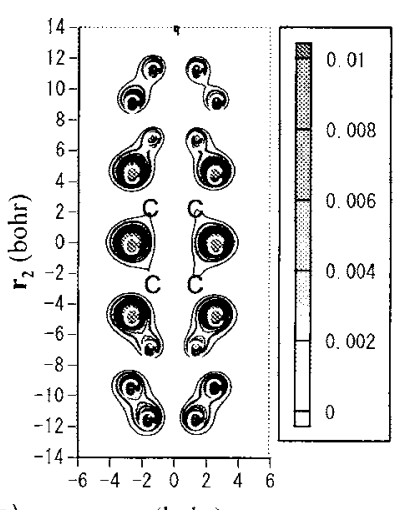

(a)
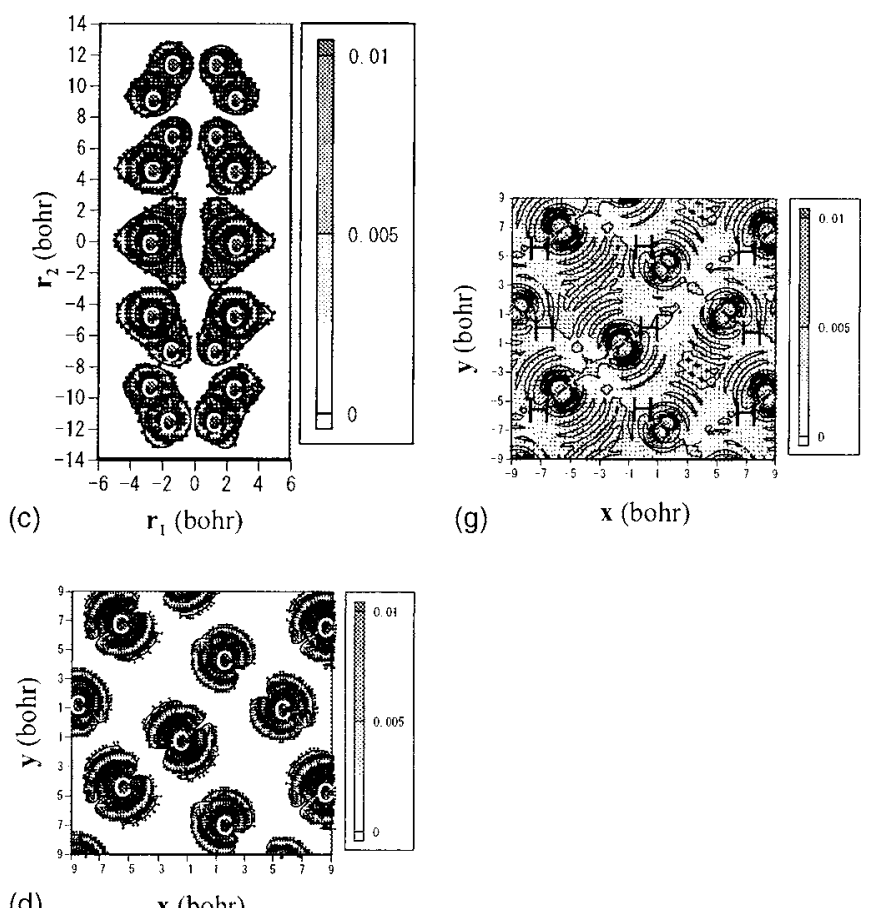

(d)

FIG. 9. Electron density, kinetic-energy density, and stress tensor density on cross sections of pentacene thin film are shown in the same way as in Fig. 8. on cross sections of molecular surface and $x y$ plane at $z=0$, respectively. (c) and (d) show kinetic-energy density by means of gray scale and tension density with vectors on the same cross sections as (a) and (b). (e) and (f) show the largest eigenvalues and their eigenvectors on cross sections of molecular surface and $x y$ plane at $z=0$, respectively, and $(\mathrm{g})$ those of only HOMO band on $x y$ plane at $z=0$.

density, the Lorentz force density, in the stationary state. Tension density resembles the centrifugal force of classical mechanics whose vector is perpendicular to the direction of motion. In this case, the electrons in $R_{D}$ are attracted by carbon atoms and packed in the $\pi$ orbitals.

This image seems to contradict the results showing effective masses lighter than the masses of free carriers in both the single crystal and the thin film. Numerous high-energy plane waves are required to represent the localized electron wave functions and the inadequacy of the basis set causes underestimation of energy-band gaps and so on. In our calculation, the plane wave basis set was not sufficient to represent the electronic states precisely and electrons tended to be delocalized to some extent, judging from the low effective masses derived from the band structures. Other calculations depend on a tight-binding method in which electron wave functions are expanded using a linear combination of atomic orbitals (LCAOs) and give results with higher effective 
masses than our results. ${ }^{33}$ LCAOs are superior for describing the localized electronic properties. There is, however, difficulty in choosing the most stable basis set.

The difference between the single crystal and the thin film is clear in their $x y$ cross sections. The electron density on the $x y$ plane is higher in the thin film shown in Fig. 9(b) than that in the single crystal shown in Fig. 8(b). The electrons in both systems, however, are localized on $\mathrm{C}$ atoms and cannot cross to the other molecules. This is further clarified by the kinetic-energy densities shown in Figs. 8(d) and 9(d). The $R_{D}$ in Fig. 9(d) occupies a larger area than that in Fig. 8(d) and this corresponds to the wider bands in the $x y$ plane in the thin film. Comparison of these two systems is valid although the carrier effective masses were estimated at small values. There is little difference in electron density and kinetic-energy density along the long axis of a pentacene molecule between the single crystal and the thin film. Figures $8(\mathrm{e})$ and $8(\mathrm{f})$ show the stress tensor density on the cross sections along the long axis of a pentacene molecule and parallel to the $x y$ plane in the single crystal, respectively, and Figs. 9(e) and 9(f) show the density in the thin film in the same way as in Figs. 8(e) and 8(f). These figures show all of the valence electrons. In these figures, covalent bonds such as $\mathrm{C}-\mathrm{C}$ bonds are represented by "tensile stress" plotted as positive values, on the other hand, electrons bound on an ion core show "compressive stress" plotted as negative values. Electrons between $\mathrm{C}-\mathrm{C}$ or $\mathrm{C}-\mathrm{H}$ cause tensile stress and their eigenvectors resemble a spindle structure. This tensile stress and spindle structure correspond to the usual image of covalent bonding very well, in which electrons are attracted from each side by cations. It should be noted that the $\pi$ character of the highest occupied molecular orbital (HOMO) bands of $\mathrm{C}$ atoms represented by positive values is hidden by the atomic compressive stress shown in Fig. 8(f) and Fig. 9(f), and therefore can only be seen in Fig. 8(g) and Fig. $9(\mathrm{~g})$. These images are the same as those of the HOMO of $\mathrm{C}_{2} \mathrm{H}_{4}$ and $\mathrm{C}_{2} \mathrm{H}_{2}$ shown in Ref. 21. As is clearly shown in Fig. $9(\mathrm{~g})$, a tight $\pi-\pi$ interaction is evidenced through a pair of adjacent pentacene molecules in the thin film, which is absent in Fig. 8(g) for the single crystal. This is the origin of the dispersion of the HOMO band of the thin film along the $\vec{b}_{1}$ direction shown in Fig. 2(b), which is not present in the case of the single crystal. The stress tensor density can show the properties of intermolecular interactions as well as intramolecular chemical bonds in detail as described above and this is an effective tool to enable discussion of chemical interactions between atoms. In this section, we introduced a way of representing the electron energy density and the stress tensor density as well as the electron density using electron wave functions, and these densities describe local electronic properties in physical and chemical terms.

\section{CONCLUSION}

We have calculated the electronic band structures of a single crystal and thin film of pentacene. A thin film was fabricated and its cell parameters were determined by x-raydiffraction analysis. ${ }^{26}$ One of three kinds of thin film model termed the $\mathrm{AB}$ orientation in this text showed a $0.44 \mathrm{eV}$ energy-band gap $\left(E_{g}\right)$ between the top of the valence band and the bottom of the conduction band. The configuration of pentacene molecules in the plane parallel to the substrate was not determined in our x-ray-diffraction analysis and two other orientations were considered in the same way as the $A B$ orientation. In the other imaginary models, $E_{g}$ shifted sensitively to $0.72 \mathrm{eV}$ in the $A^{\prime} B$ orientation and to $0.26 \mathrm{eV}$ in the $A^{\prime \prime} B$ orientation. On the other hand, $E_{g}$ of the single crystal with well-known cell parameters ${ }^{24}$ was estimated as $0.73 \mathrm{eV}$ in this work. It is concluded that the pentacene thin film has a smaller $E_{g}$ than the single crystal, and $E_{g}$ of the pentacene thin film is influenced by the configuration of pentacene molecules. Additionally, the bandwidths of the highest valence band and the lowest conduction band of the thin film were larger than those of the single crystal. These bandwidths reflect the carrier effective masses and the results of this effective mass calculation show that carrier masses in thin films tend to be lower than those in the single crystals. Our results clearly show that the electronic properties of a single crystal and thin film of pentacene are strikingly different from each other although the densities of pentacene molecules in each unit cell are not markedly different. The main reason for this difference is the orientation of pentacene molecules in the bulks.

Details of electronic interactions in pentacene solids have been analyzed in terms of kinetic-energy density, tension density, and stress tensor density as well as electron density. The regions where electrons can move freely according to classical mechanics have been clarified in terms of the kinetic-energy density and the tension density. These densities show that electrons are clearly bound on pentacene molecules and cannot cross to other molecules without quantum tunneling. Finally, the stress tensor density of the electron density has been represented and the largest eigenvalues of this density have revealed chemical-bonding properties. Electrons that contribute to intermolecular interactions as well as intramolecular covalent bonds are represented by tensile stress and show a spindle structure between carbon atoms. In this study, a representation of chemical bonding has been introduced in terms of stress tensor density derived from electron wave functions.

\section{ACKNOWLEDGMENT}

This work was supported by the Integrative IndustryAcademia Partnership (IIAP) including Kyoto University, Nippon Telegraph and Telephone Corporation, Pioneer Corporation, Hitachi, Ltd., Mitsubishi Chemical Corporation, and Rohm Co., Ltd. The authors would like to thank Dr. Y. Mori (NTT Photonics Lab.) and members of the Tachibana project group of IIAP for valuable comments and discussions. The computations were performed with the cooperation of the Academic Center for Computing and Media Studies of Kyoto University and the Research Center for Computational Science of the Okazaki Research Facilities. This paper was supported in part by the Center of Excellence (COE) for Research and Education on Complex Functional Mechanical Systems program of the Ministry of Education, Culture, Sports, Science, and Technology, Japan. 
${ }^{1}$ C. D. Dimitrakopoulos, A. R. Brown, and A. Pomp, J. Appl. Phys. 80, 2501 (1996).

${ }^{2}$ C. D. Dimitrakopoulos and P. R. L. Malenfant, Adv. Mater. (Weinheim, Ger.) 14, 99 (2002).

${ }^{3}$ C.-T. Kuo, S.-A. Chen, G.-W. Hwang, and H.-H. Kuo, Synth. Met. 93, 155 (1998).

${ }^{4}$ Y.-M. Kim, S.-W. Pyo, J.-S. Kim, J.-H. Shim, C.-H. Suh, and Y.-K. Kim, Opt. Mater. (Amsterdam, Neth.) 21, 425 (2002).

${ }^{5}$ Y. S. Lee, J. H. Park, and J. S. Choi, Opt. Mater. (Amsterdam, Neth.) 21, 433 (2002).

${ }^{6}$ J. K. Lee, J. M. Coo, S. Y. Lee, T. Y. Choi, J. Joo, J.-Y. Kim, and J.-H. Choi, Opt. Mater. (Amsterdam, Neth.) 21, 451 (2002).

${ }^{7}$ T. Minakata, I. Nagoya, and M. Ozaki, J. Appl. Phys. 69, 7354 (1991).

${ }^{8}$ T. Minakata, H. Imai, and M. Ozaki, J. Appl. Phys. 72, 4178 (1992).

${ }^{9}$ T. Minakata, H. Imai, and M. Ozaki, J. Appl. Phys. 72, 5220 (1992).

${ }^{10}$ M. Pope and C. E. Swenberg, Electronic Process in Organic Crystals and Polymers (Oxford University Press, New York, 1999), pp. 963-1004.

${ }^{11}$ V. M. Kenkre, J. D. Anderson, D. H. Dunlap, and C. B. Duke, Phys. Rev. Lett. 62, 1165 (1989).

${ }^{12}$ E. A. Silinsh and A. J. Jurgis, Chem. Phys. 94, 77 (1985).

${ }^{13}$ E. A. Silinsh, G. A. Shlihta, and A. J. Jurgis, Chem. Phys. 138, 347 (1989).

${ }^{14}$ E. A. Silinsh, A. Klimkāns, S. Lasson, and V. Čápek, Chem. Phys. 198, 311 (1995).

${ }^{15}$ M. L. Tiago, J. E. Northrup, and S. Louie, Phys. Rev. B 67, 115212 (2003).

${ }^{16}$ A. Tachibana, J. Chem. Phys. 115, 3497 (2001).

${ }^{17}$ A. Tachibana, in Stress Induced Phenomena in Metallization, edited by S. P. Baker (American Institute of Physics, New York, 2002), pp. 105-106.
${ }^{18}$ A. Tachibana, in Fundamental Perspectives in Quantum Chemistry: A Tribute to the Memory of PerOlov Löwdin, edited by E. J. Brändas and E. S. Kryachko (Kluwer Academic Publishers, Dordrecht, 2002), Vol. 2, pp. 211-239.

${ }^{19}$ A. Tachibana, in Reviews in Modern Quantum Chemistry: A Celebration of the Contributions of Robert Parr, edited by K. D. Sen (World Scientific, Singapore, 2002), Chap. 45, pp. 1327-1366.

${ }^{20}$ A. Tachinana, Int. J. Quantum Chem. 100, 981 (2004).

${ }^{21}$ A. Tachibana, J. Mol. Model. 11, 301 (2005).

${ }^{22}$ P. Ordon and A. Tachibana, J. Mol. Model. 11, 312 (2005).

${ }^{23}$ P. Ordon and A. Tachibana, J. Chem. Sci. 117, 583 (2005).

${ }^{24}$ R. B. Campbell, J. M. Robertson, and J. Trotter, Acta Crystallogr. 14, 705 (1961).

${ }^{25}$ R. B. Campbell and J. M. Robertson, Acta Crystallogr. 15, 289 (1962).

${ }^{26}$ This sample was deposited on thermally oxidized silicon wafer in a vacuum of $1.0 \times 10^{-4} \mathrm{~Pa}$ at $300 \mathrm{~K}$ of the substrate temperature. This film was grown by $500 \AA$ and the deposition rate was $0.5 \AA$ s. The structure of this sample was analyzed by $\mathrm{x}$-ray-diffraction method.

${ }^{27}$ K. Doi, K. Nakamura, and A. Tachibana, Computer code PRDFT program package, ver. 2 (Tachibana Lab. Kyoto University, Kyoto, 2004).

${ }^{28}$ D. R. Hamann, Phys. Rev. B 40, 2980 (1989).

${ }^{29}$ J. P. Perdew and Y. Wang, Phys. Rev. B 45, 13244 (1992).

${ }^{30}$ J. P. Perdew, J. A. Chevary, S. H. Vosko, K. A. Jackson, M. R. Pederson, D. J. Singh, and C. Fiolhais, Phys. Rev. B 46, 6671 (1992).

${ }^{31}$ J. M. Ziman, Principle of the Theory of Solids, 2nd ed. (Cambridge University Press, New York, 1972), pp. 183-186.

${ }^{32}$ C. Kittel, Introduction to Solid State Physics, 8 th ed. (Wiley, New York, 2005), pp. 200-208.

${ }^{33}$ A. Troisi and G. Orlandi, J. Phys. Chem. B 109, 1849 (2005). 